\title{
Long-term dissemination of acquired AmpC $\beta$-lactamases among Klebsiella spp. and Escherichia coli in Portuguese clinical settings
}

\author{
F. Freitas \& E. Machado \& T. G. Ribeiro \&
}

$\hat{A}$. Novais \& L. Peixe

\section{ABSTRACT}

We investigated the occurrence, diversity and molecular epidemiology of genes coding for acquired $\mathrm{AmpC} \beta$-lactamases ( $\mathrm{qAmpC}$ ) among clinical isolates of Enterobacteriaceae lacking inducible chromosomal AmpCs in Portugal. A total of 675 isolates non-susceptible to broadspectrum cephalosporins obtained from four hospitals and three community laboratories during a 7-year period (2002-2008) were analysed. The presence of genes coding for qAmpC was investigated by phenotypic criteria, polymerase chain reaction (PCR) and sequencing. Bacterial identification, antibiotic susceptibility testing, conjugation assays and clonal analysis were performed by standard procedures. The presence of bla qAmpC genes was detected in 50 \% (50/100; 41 Klebsiella pneumoniae, 5 Escherichia coli, 4 Klebsiella oxytoca) of the presumptive qAmpC producers. DHA-1, detected in those species, was the most prevalent qAmpC $(94 \%, 47 / 50)$, being identified since 2003 and throughout the studied period in different institutions. Despite the high clonal diversity observed, three DHA-1-producing Klebsiella spp. clones were more frequently identified. CMY-2 (6\%,3/50) was observed in B1E. coli clones. Conjugative transfer was only observed in one (2\%) CMY-2-producing isolate. Most qAmpC producers $(94 \%, 47 / 50)$ co-expressed SHV-type and/or OXA-1 or CTX-M-32 extended-spectrum $\beta$-lactamases (ESBLs). To the authors' knowledge, this is the first description of the molecular epidemiology and the long-term dissemination of qAmpCproducing Enterobacteriaceae in Portuguese clinical settings, highlighting an evolution towards a more complex epidemiological situation regarding cephalosporin resistance in Portugal. 